\title{
ADAMTS-1 inhibits angiogenesis via the PI3K/Akt-eNOS-VEGF pathway in lung cancer cells
}

\author{
Bu Wang ${ }^{1}$, Shuo Chen ${ }^{2}$, Jian-Qing Zhao ${ }^{1}$, Bao-Li Xiang ${ }^{1}$, Xin Gu ${ }^{3}$, Fang Zou ${ }^{1}$, Zhi-Hua Zhang ${ }^{1}$ \\ ${ }^{1}$ Department of Respiratory Medicine, The First Affiliated Hospital of Hebei North University, Zhangjiakou 075000, China; ${ }^{2}$ Department of \\ Respiratory Medicine, The Hospital of 81st Group Army PLA, Zhangiiakou 075000, China; ${ }^{3}$ Department of Neurology, The First Affiliated \\ Hospital of Hebei North University, Zhangjiakou 075000, China \\ Contributions: (I) Conception and design: ZH Zhang; (II) Administrative support: B Wang; (III) Provision of study materials: S Chen, JQ Zhao; \\ (IV) Collection and assembly of data: JQ Zhao; (V) Data analysis and interpretation: X Gu, F Zou; (VI) Manuscript writing: All authors; (VII) Final \\ approval of manuscript: All authors. \\ Correspondence to: Dr. Zhi-Hua Zhang. Department of Respiratory Medicine, The First Affiliated Hospital of Hebei North University, No. 12, \\ Changqing Road, Zhangjiakou 075000, China. Email: zhangzhihua015@163.com.
}

Background: ADAMTS-1 (a disintegrin and metalloproteinase with thrombospondin repeats-1) is a recently characterized protein containing a metalloproteinase domain, a disintegrin-like domain and a thrombospondin type 1 motif, which is involved in angiogenesis. However, the roles of ADAMTS-1 in angiogenesis of lung cancer (LC) remain unclear.

Methods: The mRNA expression of ADAMTS-1 and VEGF was examined by qRT-PCR. Western blots were used to detect the protein expression of ADAMTS-1 and vascular endothelial growth factor (VEGF) in A549 cells and to analyse the cellular effect of a PI3K/Akt activator and an endothelial nitric oxide synthase (eNOS) activator. ADAMTS-1 and VEGF contents in cell culture supernatants were measured by ELISA. Cell viability, cell cycle, migration, and angiogenesis of HUVECs were evaluated by MTT assay, flow cytometry, scratch assay and tube formation assay, respectively.

Results: Our data revealed that the expression of ADAMTS-1 was downregulated, while the expression of VEGF was upregulated in A549 cells. Decreased ADAMTS-1 content was also detected in A549 cell culture supernatant. Overexpression of ADAMTS-1 inhibited VEGF expression and A549 cell proliferation. Moreover, ADAMTS-1 overexpression repressed proliferation, migration and angiogenesis of HUVECs. Mechanistically, ADAMTS-1 suppressed the expression of VEGF in HUVECs by inhibiting PI3K/AkteNOS, while a PI3K activator and an eNOS activator each partly reversed the expression of VEGF. In addition, activation of the PI3K/Akt pathway or VEGF overexpression reversed the inhibitory effect of ADAMTS-1 overexpression on HUVECs angiogenesis.

Conclusions: These results indicated that ADAMTS-1 inhibited angiogenesis of LC cells via regulation of the PI3K/Akt-eNOS/VEGF axis, which shed light on LC pathogenesis and provided potential targets for LC therapy.

Keywords: ADAMTS-1; PI3K/Akt-eNOS; vascular endothelial growth factor (VEGF); angiogenesis; lung cancer (LC)

Submitted May 06, 2019. Accepted for publication Oct 08, 2019.

doi: $10.21037 /$ tcr.2019.10.34

View this article at: http://dx.doi.org/10.21037/tcr.2019.10.34 


\section{Introduction}

Lung cancer (LC) is the leading cause of cancer-related deaths in the world (1). Its 5 -year survival rate after diagnosis is only $15.9 \%$, which is only slightly improved over the past decades (2). Due to the lack of typical symptoms and signs, the majority of LC patients have commonly been diagnosed at advanced stages, usually leading to poor outcomes during surgery or chemotherapy (3). LC depends on angiogenesis throughout its initiation, development and metastases, which is typical of angiogenesis-dependent disease $(4,5)$. Therefore, a new target for regulating angiogenesis would provide new therapeutic opportunities for LC.

Angiogenesis is the formation of new blood vessels, which involves the migration, growth, and differentiation of endothelial cells (6). Evidence has shown that angiogenesis is a relatively early event in the development of cancer. In invasive tumours, the balance between apoptosis and proliferation depends on the ability of tumours to induce neovascularization. Early in the late 1960s, there was evidence indicating that tumour angiogenesis was mediated by secreted factors released into circulation cancer cells (7). Angiogenic squamous dysplasia (ASD) is a lesion of the capillary ring that presents as a histologically abnormal bronchial epithelium and has been observed in precancerous lesions in individuals at high-risk for developing LC (8).

Although many possible angiogenesis regulators have been identified, vascular endothelial growth factor (VEGF) has particularly strong involvement in tumourrelated angiogenesis (9). As a strong promoter of angiogenesis, VEGF contributes to the endothelial and vascular infiltration of new vessels through the interaction of VEGF-A and VEGFR-2 (10). These pathways together promote endothelial cell survival, proliferation, migration, invasion and tube formation, thus forming a new vascular network (11). Previous studies demonstrated that ADMATS-1 reduces the proliferation, invasion and migration of angiogenesis that results from the interplay between VEGF and FGF-2 $(12,13)$. Recent studies confirmed that ADAMTS-1 has low expression levels in LC cells (14). However, the molecular mechanism of ADAMTS- 1 in LC remains unclear.

In this study, we hypothesized that ADAMTS-1 inhibited the proliferation, migration and angiogenesis of HUVECs through regulation of the PI3K/Akt-eNOS-VEGF pathway. The present study used a series of experiments to test this hypothesis.

\section{Methods}

\section{Cell culture}

The cell lines BEAS-2B and A549 were provided by the Korean Cell Line Bank. Cells were maintained in Dulbecco's modified Eagle's medium (DMEM, Grand Island) with 10\% fetal bovine serum (FBS) supplemented with penicillin and streptomycin (Beyotime, Shanghai, China), and they were kept at $37{ }^{\circ} \mathrm{C}$ in a humidified atmosphere with $95 \%$ air and $5 \% \mathrm{CO}_{2}$ until use. HUVECs were purchased from Sciencell Co., Ltd., seeded at 60-70\% confluence and incubated with the mixed medium as described below. The HUVECs were cultured in a cell incubator that was maintained at $5 \% \mathrm{CO}_{2}$ and $37^{\circ} \mathrm{C}$.

\section{Co-culture system}

A549 cells were seeded into the wells of 6-well plates at $1.5 \times 10^{6}$ cells per well, and HUVECs were seeded at $1.5 \times 10^{6}$ cells per well in a transwell chamber of a 6 -well plate containing a $0.4 \mu \mathrm{m}$ porous membrane. Cells were cultured in DMEM with $10 \%$ FBS or high-glucose (HG) DMEM with $10 \%$ FBS. After six hours, the cells adhered to the PDMS membrane. Then, medium with $10 \%$ FBS was used to replace plating medium, which was followed by incubation for $12 \mathrm{~h}$; then, DMEM with $2 \%$ FBS replaced that medium and was followed by 8 more hours of incubation. The percentage of FBS was decreased in A549 cells co-cultured with HUVECs at decreasing increments of $1 \%$. Cell samples were harvested after 3 days of co-culture. The co-culture system was also used to study the effect of A549 cells on HUVEC migration. After $24 \mathrm{~h}$ of incubation, the cells were washed with phosphate-buffered saline (PBS), fixed with $4 \%(\mathrm{w} / \mathrm{v})$ polyformaldehyde, and stained with 4',6-diamino2-benzoindole (DAPI) for 15 minutes, and the number of cells in the lower chamber was counted. The degree of cell migration was calculated over 5 microfields per well and is expressed as the average number of cells in each microfield.

\section{$R N A$ extraction and quantitative real-time PCR}

Total RNA was isolated using TRIzol (Invitrogen, Carlsbad, CA, USA). Then, equal amounts of RNA $(1 \mu \mathrm{g})$ were reverse transcribed into cDNA using the PrimeScript RT Master Mix Perfect Real Time Kit (TaKaRa, Dalian, China). Real-time PCR was performed using cDNA 
and a SYBR Green (TaKaRa, Dalian, China) detection system, which included 40 cycles of amplification. Primer sequences for detection of ADAMTS-1 and VEGF mRNA expression were as follows: ADAMTS-1-F: 5'-AAGCTGCTGATGGCACATATATTCA-3' and ADAMTS-1-R: 5'-TTTTAGGTCGAAGGGCATTGC-3'; VEGF-F: 5'-TGCATTCACATTTGTTGTGC-3' and VEGF-R: 5'-AGACCCTGGTGGACATCTTC-3' (15). The primers were synthesized by Shanghai Sangon Co., Ltd. Gene expression levels were calculated relative to the housekeeping gene GAPDH.

\section{Western blot analysis}

Cell proteins were extracted using a Total Protein Extraction Kit (Keygentec, China). Protein concentrations were determined by the bicinchoninic acid (BCA) method (Beyotime Biotechnology, Shanghai, China). A total of $20 \mu \mathrm{g}$ of protein per sample was separated by $10 \%$ SDSPAGE and transferred to a PVDF membrane (Millipore, Darmstadt, Germany). Then, the membranes were blocked in $5 \%$ non-fat milk and incubated at $4{ }^{\circ} \mathrm{C}$ overnight with anti-ADAMTS-1 (1:1,000, Cell Signaling Technology, USA), anti-PI3K (1:1,000, Cambridge, MA, USA), antip-PI3K (1:1,000, Abcam, MA, USA), anti-Akt (1:1,000, Abcam, MA, USA), anti-p-Akt (1:1,000, Abcam, MA, USA), anti-eNOS (1:200, Santa Cruz Biotechnology, Santa Cruz, CA, USA), anti-p-eNOS (1:200, Santa Cruz Biotechnology, Santa Cruz, CA, USA), anti-VEGF (1:1,000, Abcam, MA, USA), or anti-GAPDH antibody (1:5,000, Abcam, MA, USA). Subsequently, the membranes were washed with TBST and then incubated with a secondary antibody (HRP-conjugated goat anti-rabbit IgG $(1: 2,000)$, Abcam, MA, USA) at room temperature for $1 \mathrm{~h}$. Specific antibody binding was detected using an ECL detection kit (Pierce Biotechnology, Rockford, IL, USA).

\section{Plasmid construction and cell transfection}

The coding regions of ADAMTS-1 were amplified by PCR . Then, the PCR products were cloned into the pcDNA3.1 plasmid to create a new plasmid named pcDNA3.1ADAMTS-1 (Beyotime, Shanghai, China). A549 cells were seeded into 6-well plates and incubated for $24 \mathrm{~h}$. Then, $1,000 \mathrm{ng}$ of pcDNA3.1 or pcDNA-ADAMTS-1 plasmid was transfected into A549 cells according to the manufacturer's instructions using Lipofectamine ${ }^{\mathrm{TM}} 2000$ (Invitrogen). Then, the supernatant from the 6-well plates was mixed with DMEM at a 1:1 ratio. HUVECs were cultured with the mixed medium at $5 \% \mathrm{CO}_{2}$ and $37{ }^{\circ} \mathrm{C}$. Subsequently, the proliferation, migration and angiogenesis of HUVECs were evaluated. HUVECs were treated with the PI3K activator 740Y-P (S786501, Selleck, Shanghai, China) or the eNOS activator calcium ionophore A23187 (C9275, Sigma, Shanghai, China). After 24 h, the protein levels of key genes in the PI3K/Akt-eNOS-VEGF pathway in HUVECs were determined by Western blot.

\section{Enzyme linked immunosorbent assay (ELISA)}

The ADAMTS-1 and VEGF levels in cell culture supernatants were detected using the Human ADAMTS1 ELISA Kit (ab213751, Abacam) and Human VEGF ELISA Kit (ab222510, Abcam) respectively, according to the manufacturer's instructions. Briefly, cell culture supernatants were centrifuged at 2,000 $\mathrm{g}$ to remove debris, and the supernatants were diluated with Sample Diluent NS + 2X Enhancer, added in to 96 -well plate $(50 \mu \mathrm{L} /$ well $)$, incubated with $50 \mu \mathrm{L}$ Antibody Cocktail for $1 \mathrm{~h}$ at room temperature, incubated with $100 \mu \mathrm{L}$ TMB Substrate for $10 \mathrm{~min}$ in darkness, and finally mixed with $100 \mu \mathrm{L}$ Stop Solution. The protein contents in supernatants were determined by measuring the absorbance at $450 \mathrm{~nm}$ (OD450) using a plate reader.

\section{Proliferation assays}

HUVEC viability was evaluated by MTT assay using linear curves plotted as described before (16). Briefly, equal amounts of HUVECs were seeded into a 96-well plate. After $24 \mathrm{~h}$, the cells were treated with $20 \mu \mathrm{L}$ of $5 \mathrm{mg} / \mathrm{mL}$ MTT and incubated for $3.5 \mathrm{~h}$ at $37^{\circ} \mathrm{C}$, which was followed by the addition of $150 \mu \mathrm{L}$ of MTT solvent for $15 \mathrm{~min}$. Finally, the absorbance was read at $590 \mathrm{~nm}$ with a reference filter of $620 \mathrm{~nm}$.

\section{Cell cycle analysis}

Flow cytometry analysis was performed to determine the cell cycle. Cells were fixed in cold $70 \%$ ethanol for $30 \mathrm{~min}$ at $4{ }^{\circ} \mathrm{C}$. After washing, cells were treated with $50 \mu \mathrm{L}$ of a $100 \mu \mathrm{g} / \mathrm{mL}$ stock of RNase (EN0531, Thermo, USA). Then, the cells were incubated with propidium iodide for $5 \mathrm{~min}$. G0/G1 and S phase cell numbers were acquired by flow cytometry and analysed using FlowJo software version 8.8 (Tree Star, Inc., Ashland, OR, USA). 

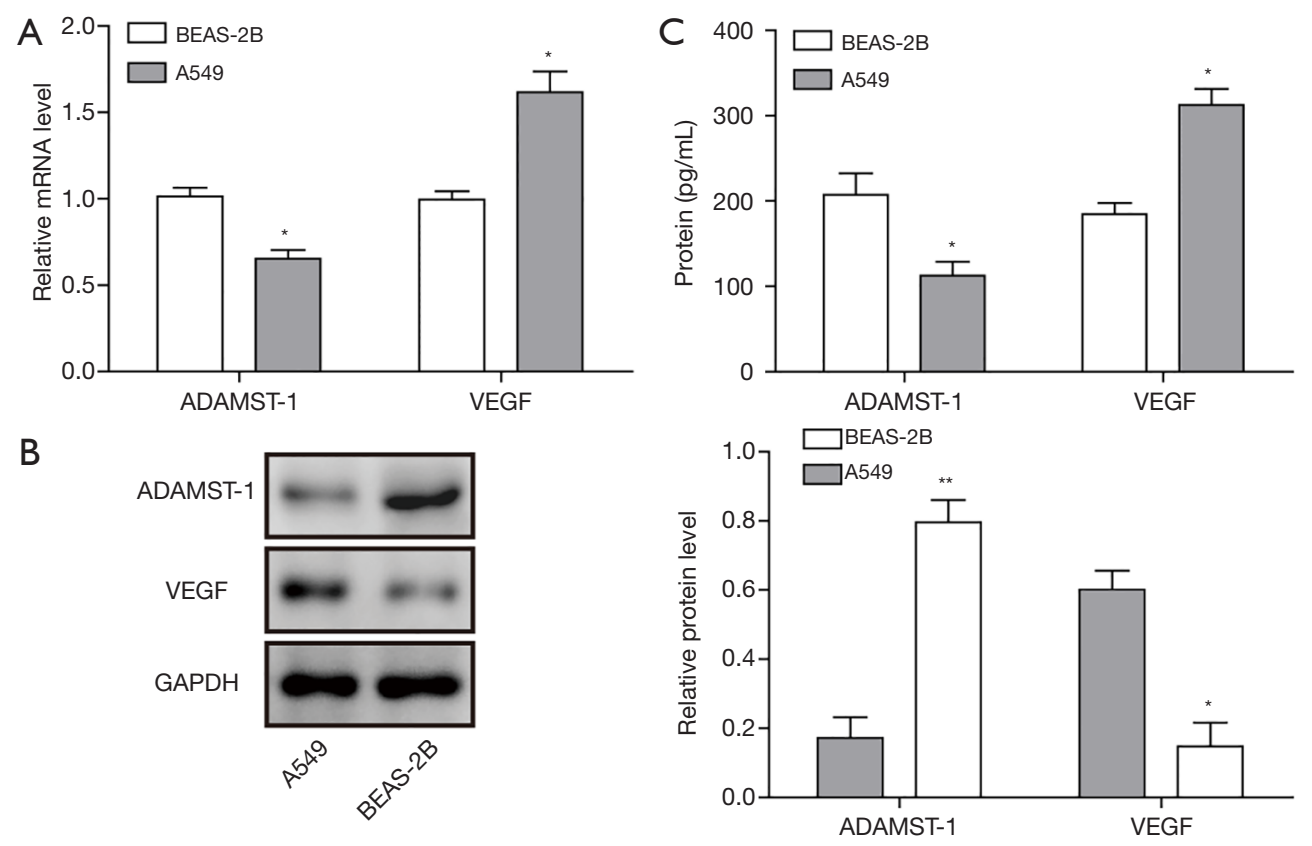

Figure 1 ADAMTS-1 was lowly expressed while VEGF was highly expressed decreased in A549 cells. (A) ADAMTS-1 and VEGF mRNA levels in A549 and BEAS-2B cells were examined by qRT-PCR; (B) ADAMTS-1 and VEGF protein abundances in A549 and BEAS-2B cells were determined by Western blotting; (C) ADAMTS-1 and VEGF contents in the culture supernatants of A549 and BEAS-2B cells by ELISA. ${ }^{*} \mathrm{P}<0.05 ;{ }^{* *} \mathrm{P}<0.01$. VEGF, vascular endothelial growth factor; ELISA, enzyme linked immunosorbent assay.

\section{Scratch assay}

Cell migration was determined by cell scratch assay. HUVECs were seeded in the wells of 6 -well plates and incubated with $1 \mu \mathrm{g} / \mathrm{mL}$ of mitomycin $\mathrm{C}$ for $1 \mathrm{~h}$. Using sterile toothpicks, a "+" was drawn on the cell surface. The wells were rinsed with PBS 2-3 times to wash away floating cells and then serum-free medium was added back to the wells. The culture was continued under $5 \% \mathrm{CO}_{2}$ and saturated humidity (set as $0 \mathrm{~h}$ ). The migration of the cells was photographed under an inverted microscope at 0 and 24 h. ImageJ (NIH Image, Bethesda, MD, USA) was used to measure the scratch width, and the cell migration distance between each group was calculated.

\section{Angiogenesis assay}

Tumour endothelial cell matrix Matrigel $(0.1 \mathrm{~mL})$ was polymerized on $24-w e l l$ plates, and HUVECs $\left(5 \times 10^{4}\right.$ cells/well $)$ were plated onto Matrigel pads with complete growth medium. The Matrigel assay was used to assess the spontaneous formation of capillary-like structures in vitro (17). After $24 \mathrm{~h}$, the formed vessel tubes were photographed under a microscope with a Nikon camera (Nikon, Japan). HUVECs were also collected for Western blot analysis.

\section{Statistics}

All of the experiments were performed in triplicate. Error bars represent the mean \pm SD. Data were analysed by oneway ANOVA, followed by the Newman-Keuls post hoc test. Student's $t$-tests were used for paired comparisons. $\mathrm{P}<0.05$ was considered statistically significant.

\section{Results}

\section{ADAMTS-1 and VEGF expression in LC cells}

To determine the expression of ADAMTS-1 and VEGF in LC cells, we used qRT-PCR and Western blotting to detect their mRNA and protein levels in the normal human bronchial epithelial cell line BEAS-2B and the LC cell line A549. The results demonstrated that the expression of ADAMTS- 1 was significantly downregulated, while VEGF was upregulated in the A549 LC cells compared with BEAS-2B cells (Figure 1A,B). 
Moreover, we also detected the expression of ADAMTS-1 and VEGF by ELISA, and found that ADAMTS-1 levels in A549 cell culture supernatant was significantly lower than that of the BEAS-2B cells, while VEGF expression in A549 cell culture supernatant was greatly higher than that of the BEAS-2B cells (Figure 1C). These results revealed that ADAMTS-1 expression was increased while VEGF expression was decreased in A549 cells.

\section{ADAMTS-1 overexpression repressed VEGF expression and inbibited A549 cell proliferation}

To investigate the proliferation and migration of HUVECs, pcDNA3.1 and pcDNA3.1-ADAMTS-1 were transfected into A549 cells. As shown in Figure 2A,B, ADAMTS-1 mRNA and protein levels were enhanced dramatically in A549 cells after transfection with pcDNA3.1-ADAMTS-1. This result means that the ADAMTS1 expression plasmid was constructed successfully and that ADAMTS1 was highly expressed in A549 cells. Also, we observed that both the mRNA and protein levels of VEGF in A549 cells were significantly decreased by transfection with pcDNA3.1ADAMTS-1 plasmids (Figure 2C,D). Remarkable decrease of VEGF content in supernatants of A549 cells transfected with pcDNA3.1-ADAMTS-1 was also detected by ELISA (Figure 2E). Moreover, our MTT assay showed that ADAMTS-1 overexpression significantly suppressed the proliferation of A549 cells, compared with the control group and those transfected with the empty plasmids (Figure $2 F$ ). These results showed that ADAMTS-1 overexpression could repress VEGF expression and A549 cell proliferation.

\section{ADAMTS-1 overexpression inbibits proliferation, migration and angiogenesis of HUVECs}

To explore the influence of ADAMTS-1 on HUVEC cell functions, the supernatants of A549 cell with or without ADAMTS-1 overexpression were then used to culture the HUVEC cells. Our MTT assay showed that the proliferation of HUVEC cells cultured with supernatants of pcDNA3.1-ADAMTS-1-transfected A549 cells was significantly decreased compared with the pcDNA3.1 transfected control group (Figure 3A). Flow cytometry was performed to analyse the distribution of the cell cycle, and the results indicated that HUVEC cell cycle was significantly repressed by culturing with supernatants of A549 cells transfected with pcDNA3.1-ADAMTS-1
(Figure $3 B$ ). There were fewer cells in the $S$ phase, but there were more cells in the G0/G1 phase in the pcDNA3.1ADAMTS-1 transfection group compared to the pcDNA3.1 transfection group. These data suggested that ADAMTS-1 overexpression inhibited HUVEC proliferation. Moreover, our scratch assays showed that compared with the pcDNA3.1 transfection control group, the HUVECs in the pcDNA3.1-ADAMTS-1 transfection group had significantly decreased migration speed (Figure 3C). Next, we evaluated the role of ADAMTS-1 in angiogenesis by angiogenesis assays. Our results showed that HUVEC tube formation was significantly inhibited in the pcDNA3.1ADAMTS-1 transfection group, compared with the pcDNA3.1 transfection group (Figure 3D). This indicated that overexpression of ADAMTS-1 significantly inhibited HUVEC proliferation, migration and angiogenesis.

\section{ADAMTS-1 downregulates VEGF via inbibiting PI3K/ Akt-eNOS}

Since VEGF is a strong promoter of angiogenesis, the effect of ADAMTS-1 on VEGF signalling was further investigated. Western blot assays showed that overexpression of ADAMTS-1 significantly decreased p-PI3K, p-Akt, p-eNOS levels and the expression of VEGF in HUVECs. However, 740Y-P reversed the p-PI3K, p-Akt, p-eNOS and VEGF levels by activating PI3K, and A23187 reversed the expression of $\mathrm{p}$-eNOS and VEGF in HUVECs by activating downstream eNOS. Along with the increased amounts of phosphorylated PI3K/Akt/eNOS, the protein level of VEGF in HUVECs was also increased (Figure 4). Our results revealed that ADAMTS-1 suppressed the expression of VEGF via inhibiting the phosphorylation of PI3K, Akt and eNOS.

\section{ADAMTS-1 inbibits the proliferation, migration and angiogenesis of HUVECs through the PI3K/Akt-eNOS- VEGF patbway}

During the process of angiogenesis, VEGF activation is mediated by the PI3K/Akt pathway (18). We examined whether ADAMTS-1 affected cell proliferation, migration and angiogenesis through the PI3K/Akt-eNOS-VEGF pathway. The MTT assay results showed that treatment with the PI3K activation peptide (740Y-P) partially restored the inhibition of pcDNA3.1-ADAMTS-1 on the proliferation of HUVECs (Figure 5A). Also, we overexpression VEGF in 
A

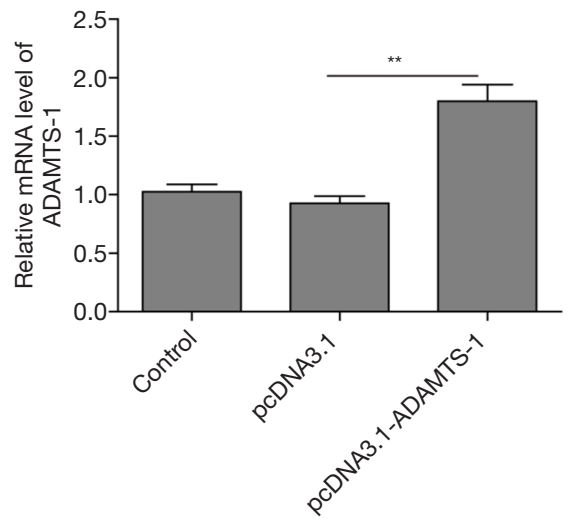

C

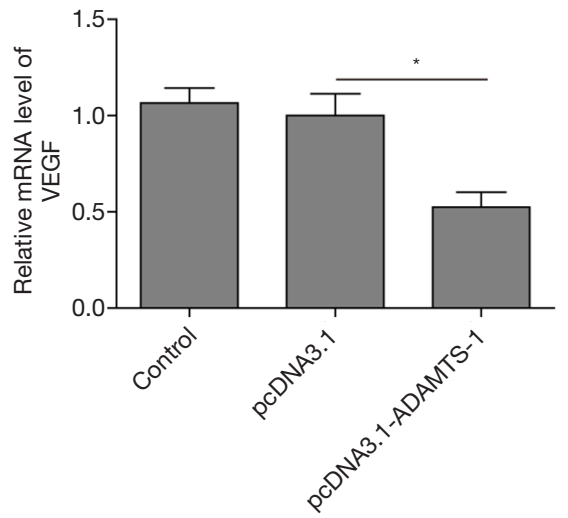

E

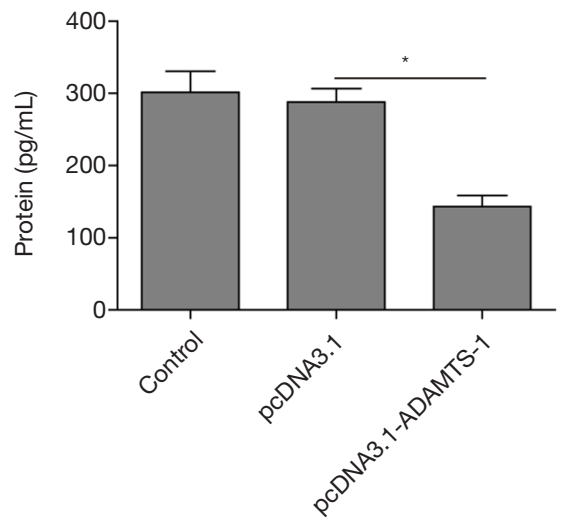

B

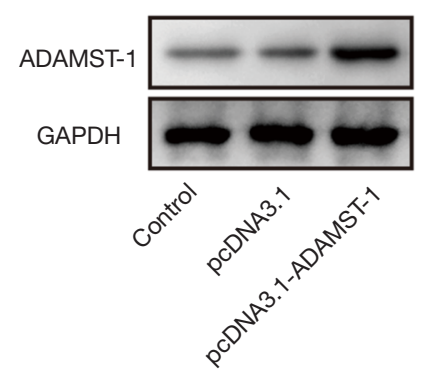

D

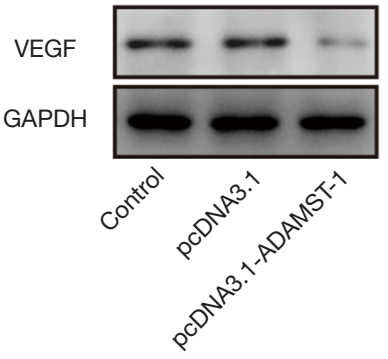

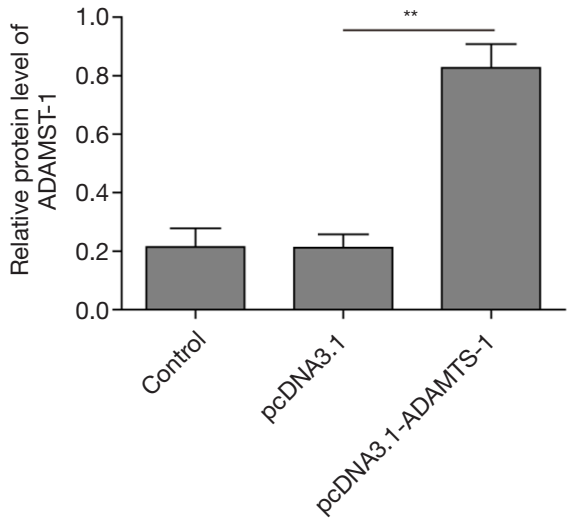

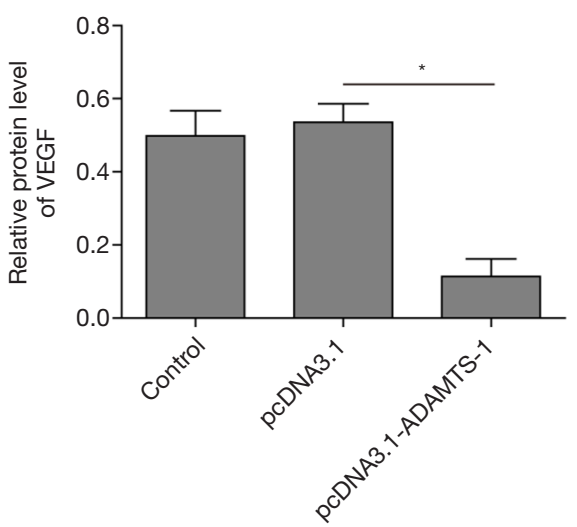

$\mathrm{F}$

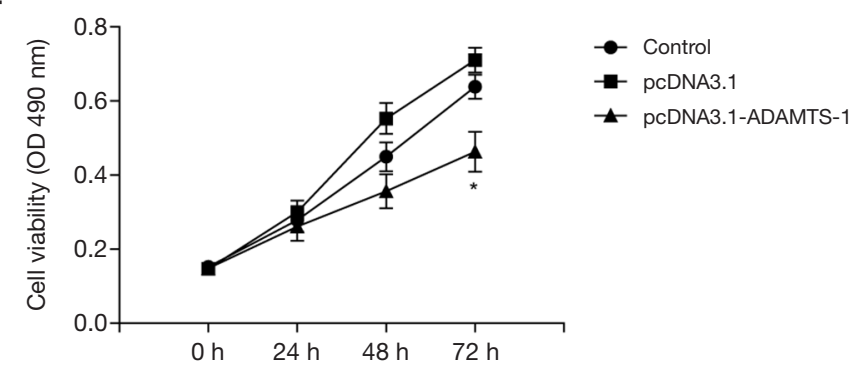

Figure 2 Overexpression of ADAMTS-1 repressed VEGF expression and inhibited A549 cell proliferation. A549 cells were transfected with pcDNA3.1-ADAMTS-1 or pcDNA3.1. (A) The mRNA level of ADAMTS-1 in A549 cells was examined by qRT-PCR; (B) the protein level of ADAMTS-1 in A549 cells was examined by Western blotting; (C) relative VEGF mRNA levels in A549 cells with ADAMTS-1 overexpression detected by qRT-PCR; (D) the VEGF protein levels in A549 cells with ADAMTS-1 overexpression analysed by western blotting; (E) the VEGF protein levels in A549 cells with ADAMTS-1 overexpression detected by ELISA; (F) the viability of A549 cells with ADAMTS-1 overexpression by MTT assay. ${ }^{*} \mathrm{P}<0.05 ;{ }^{*} \mathrm{P}<0.01$. VEGF, vascular endothelial growth factor.

HUVECs by transfection with pcDNA3.1-VEGF plasmids, and found that VEGF overexpression promoted HUVEC proliferation and mitigated the suppression of HUVEC proliferation induced by supernatants from ADAMTS- 1-overexpressing A549 cells (Figure 5A). At the same time, cell scratch assays showed that the inhibitory effect of ADAMTS-1 on HUVECs migration can be partially reversed by 740Y-P treatment and VEGF overexpression 

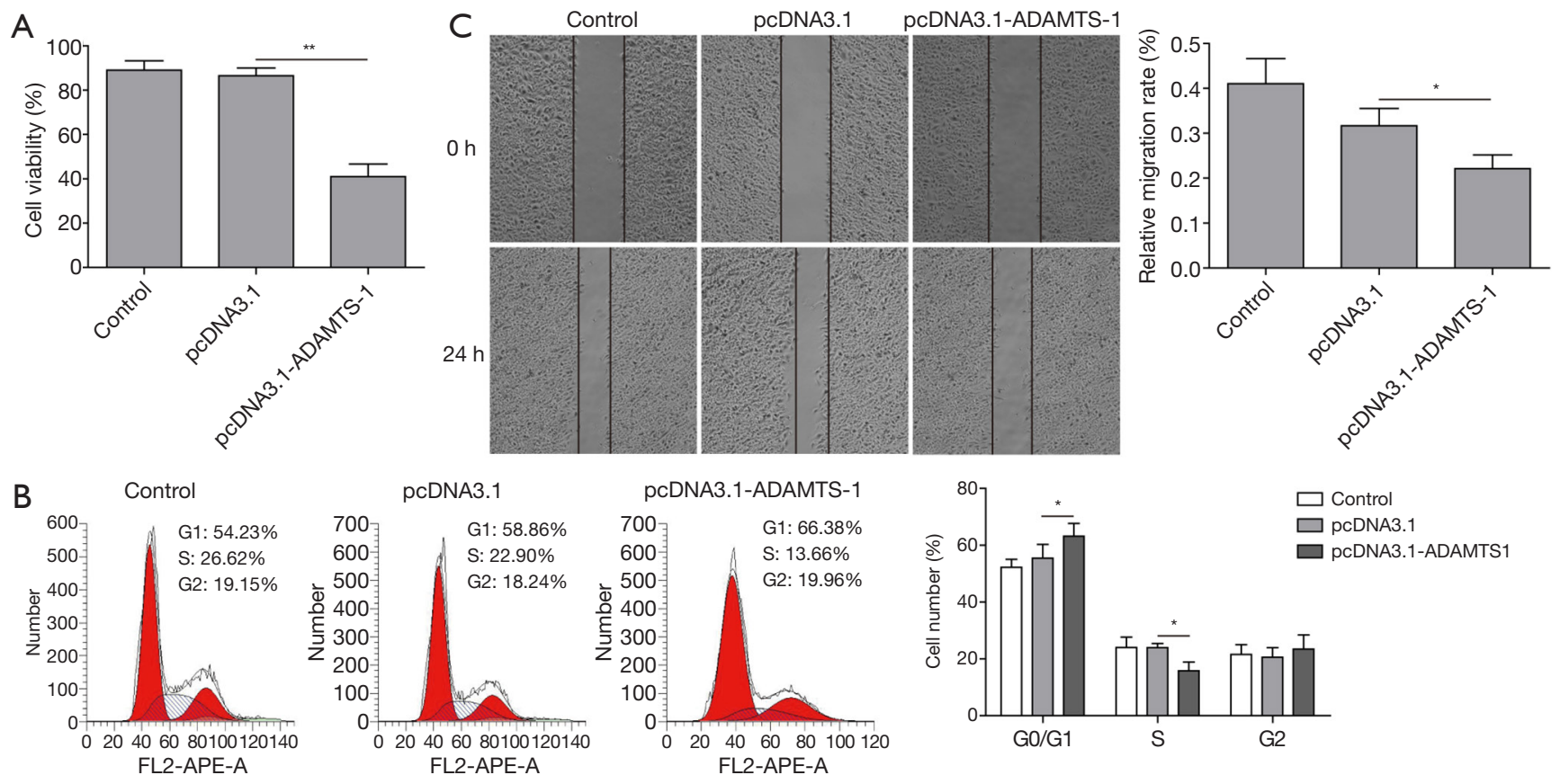

D
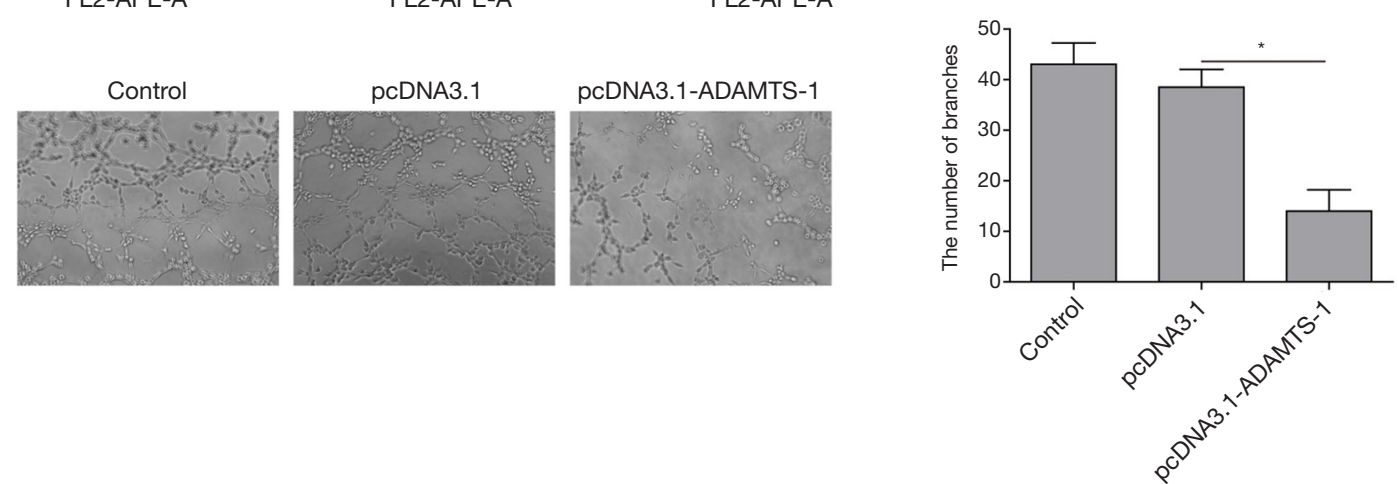

Figure 3 ADAMTS-1 inhibited in vitro angiogenesis of HUVECs. The supernatants of A549 cells with or without ADAMTS-1 overexpression were used to culture HUVECs. (A) Cell viability was analysed by MTT assay in HUVECs cultured with the supernatant from the pcDNA3.1-ADAMTS-1 transfection group or the pcDNA3.1 transfection group; (B) HUVEC cell-cycle distribution (S phase and G0/G1 phase) was analysed by flow cytometry; (C) scratch assays were performed to detect HUVEC cell migration; (D) tube formation of HUVECs was detected by angiogenesis assays. ${ }^{*} \mathrm{P}<0.05 ;{ }^{* *} \mathrm{P}<0.01$.

in HUVECs (Figure 5B,C). Moreover, the formation of HUVEC tubes increased in the pcDNA3.1-ADAMTS-1 transfection group treated with 740Y-P compared with the untreated pcDNA3.1-ADAMTS-1 transfection group (Figure 5D,E). And VEGF overexpression in HUVECs also abrogated the suppression of HUVEC tube formation caused by ADAMTS-1 overexpression in A549 cells (Figure 5D,E). These results indicate that ADAMTS-1 inhibited the proliferation, migration and angiogenesis of HUVECs through the PI3K/Akt-eNOS-VEGF pathway.

\section{Discussion}

Worldwide, the number of patients with LC increases annually by more than 1.6 million (19). Most patients are at an advanced stage when they are first diagnosed. Some reports indicate that VEGF-regulated blood vessel extension, maintenance and remodelling are the main causes of invasion and metastasis in LC patients. (20). Tumour blood vessel growth depends on angiogenesis. When the tumour grows to $1-2 \mathrm{~mm}^{3}$, oxygen and nutrients must be 

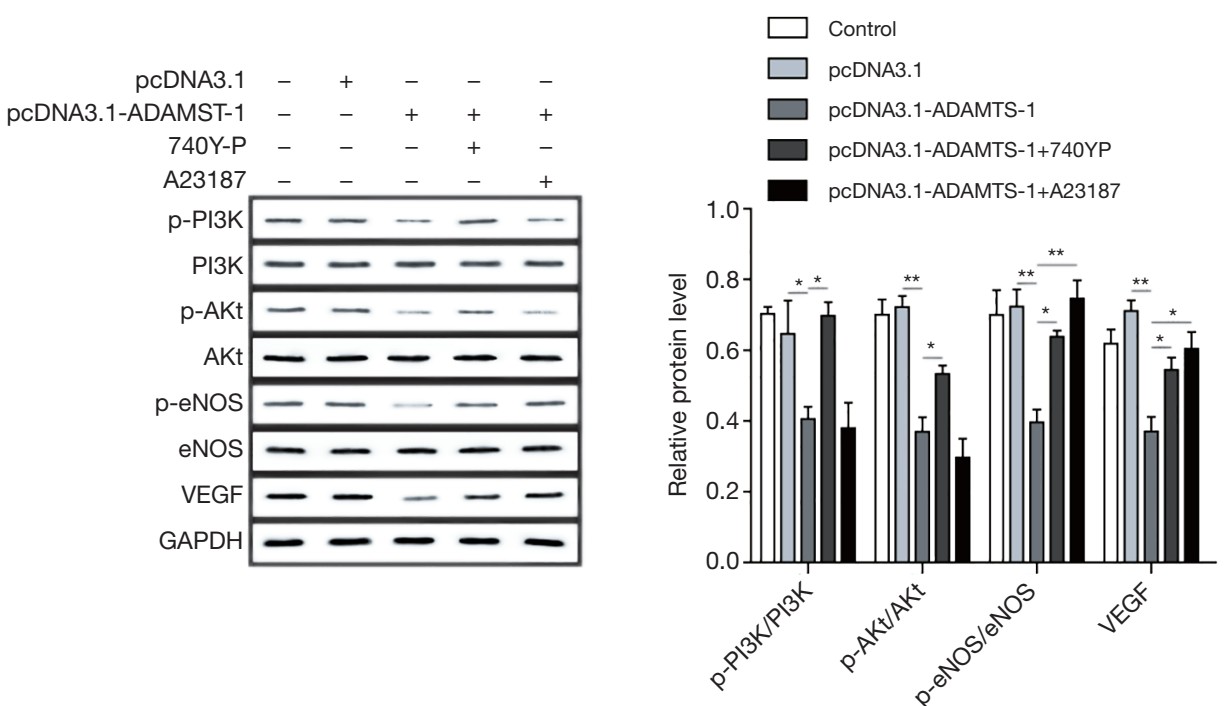

Figure 4 ADAMTS-1 downregulated the PI3K/Akt-eNOS-VEGF signalling pathway in HUVECs. HUVECs cultured with the supernatant from the pcDNA3.1-ADAMTS-1 transfection group or the pcDNA3.1 transfection group were incubated for 24 h with $740 Y-P$ or A23187, which was followed by Western blot analysis. ${ }^{*} \mathrm{P}<0.05 ;{ }^{* *} \mathrm{P}<0.01$. VEGF, vascular endothelial growth factor.

provided by the generation of new blood vessels. Therefore, control of angiogenesis has emerged as an important mechanism for suppressing tumour growth. In this study, we found that ADAMTS-1 inhibited the proliferation, migration, and angiogenesis of HUVECs through the PI3K/Akt-eNOS-VEGF pathway.

ADAMTS-1 was the first identified member of the ADAMTS family. Production of ADAMTS-1 is modulated in LC, breast cancer, pancreatic cancer, and fibrosarcoma $(21,22)$, and the fragment of ADAMTS-1 containing the TSP-1 motif can exhibit antitumour activity (23). In the present study, we reported that expression of ADAMTS-1 was downregulated, while VEGF was upregulated in A549 cells. Further, overexpression of ADAMTS-1 suppressed VEGF expression in A549 cells, which caused significantly repressed A5 49 proliferation. Also, ADAMTS-1 overexpression inhibited the proliferation and migration of HUVECs. Some reports note the inhibitory effects of ADAMTS-1 on angiogenesis that we observed. For instance, a previous study confirmed that ADAMTS-1 disrupted microtumour formation of fibrosarcoma through HGF/c-MET signalling (24) and inhibited endothelial cell proliferation by suppressing FGF (25). Masanari et al. reported that ADAMTS-1 gene transfer inhibited angiogenesis in vitro and in vivo, probably due to ADAMTS-1-induced apoptosis of endothelial cells (26). In addition, Gustavsson et al. found that ADAMTS-1 might have anti-angiogenic and antimetastatic roles in hormonerefractory prostate cancer, where low ADAMTS1 expression was associated with a high MVD and metastasis (27). All of the results above indicate that ADAMTS-1 has antiangiogenic effects on LC cells.

VEGF is an important regulator of angiogenesis; upon activation, VEGF promotes angiogenesis in human lung tumour associated-endothelial cells (28). Intracellular tyrosine kinases, for instance, PI3K/Akt and eNOS, were activated once VEGF bound to the VEGF receptor on the surface of endothelial cells (29). Akt can promote eNOS phosphorylation and NO release (30,31). Released NO can activate its downstream pathways and increase the expression of VEGF in endothelial cells (32). Cell survival is very important during angiogenesis. A major pathway through which growth factors promote endothelial cell survival is the PI3K-dependent activation of the antiapoptotic kinase Akt/PKB (33). Some reports found that ADAMTS-1 functions as an inhibitor of VEGFR2 phosphorylation by binding to VEGF165 (34). Chen et al. demonstrated that overexpression of ADAMTS- 1 had angio-inhibitory effects on primary gastric cancer because it decreased VEGF gene expression (15). In line with a previous study, our results demonstrated that ADAMTS-1 suppressed the expression of the VEGF gene in both A549 and HUVEC cells by inhibiting the PI3K/Akt-eNOS pathway. The proliferation of A549 cells could be repressed 
A

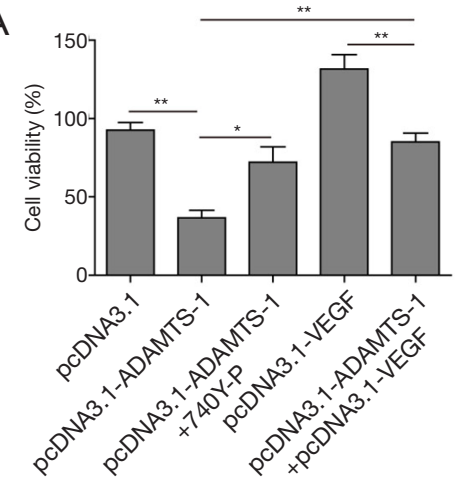

C

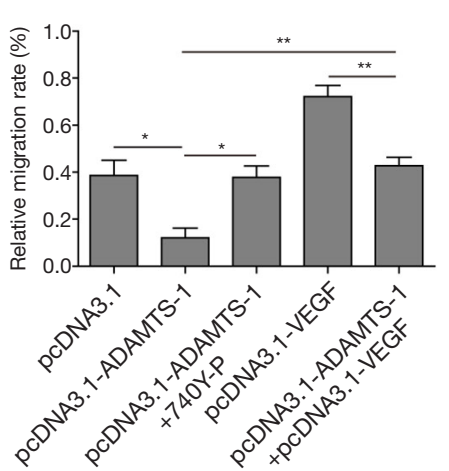

E
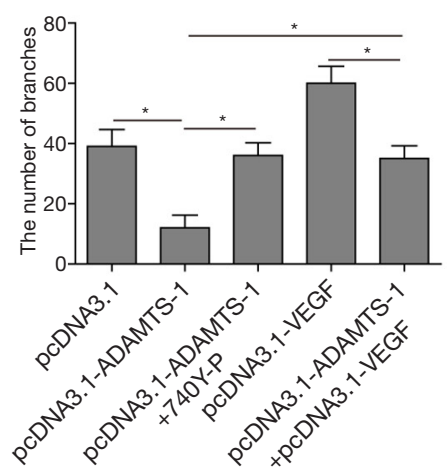

B
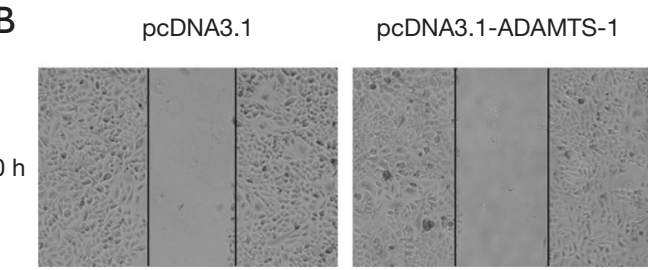

pcDNA3.1-ADAMTS-1
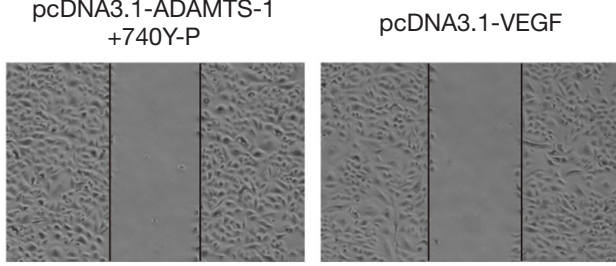

pcDNA3.1-ADAMTS-1 +pcDNA3.1-VEGF
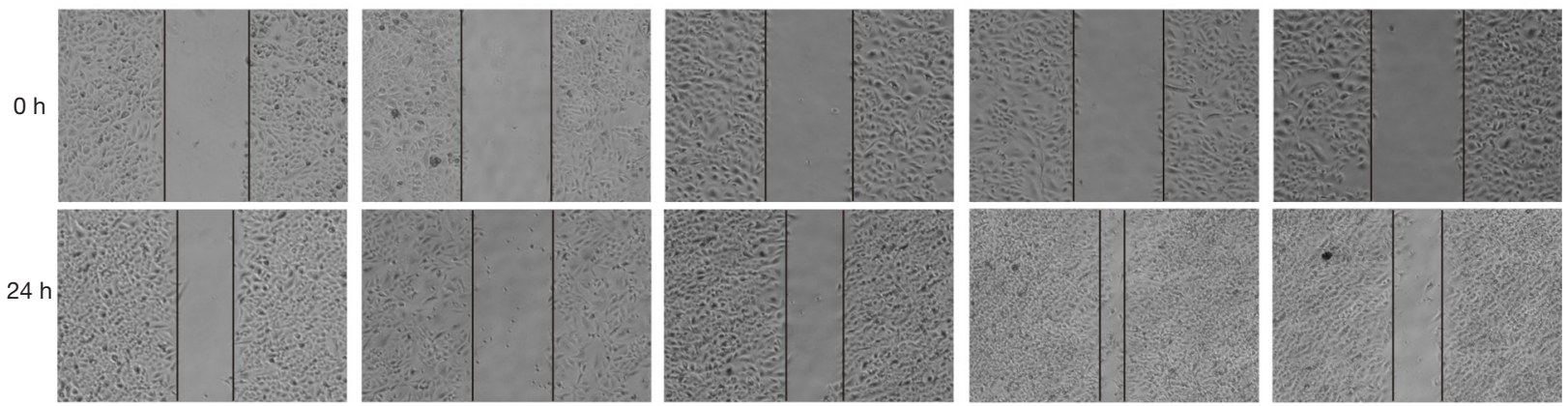

pcDNA3.1-ADAMTS-1 $+740 Y-P$

pcDNA3.1-VEGF
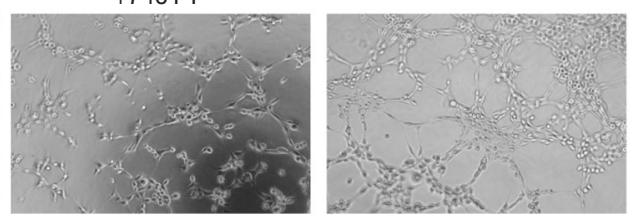

pcDNA3.1-ADAMTS-1 +pcDNA3.1-VEGF
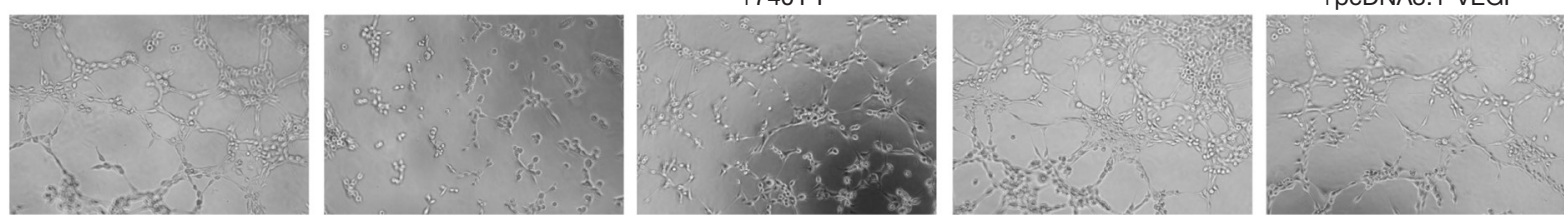

Figure 5 ADAMTS-1 inhibited the proliferation, migration and angiogenesis of HUVECs through the PI3K/Akt-eNOS-VEGF pathway. A549 cells were transfected with pcDNA3.1-ADAMTS-1 or pcDNA3.1. HUVECs were transfected with the pcDNA3.1-VEGF. (A) Effects of 740Y-P and VEGF overexpression on the proliferation of HUVECs cultured with supernatants from ADAMTS-1-overexpression A549 cells. Cell proliferation was examined by MTT assay; (B,C) influences of 740Y-P and VEGF overexpression on the migration of HUVECs cultured with supernatants from ADAMTS-1-overexpression A549 cells. Scratch assays were performed to measure cell migration; (D,E) regulation of HUVEC tube formation by 740Y-P and VEGF overexpression after being cultured with supernatants from ADAMTS-1overexpression A549 cells. HUVEC tube formation was detected by angiogenesis assay. ${ }^{*} \mathrm{P}<0.05 ;{ }^{* *} \mathrm{P}<0.01$. VEGF, vascular endothelial growth factor.

by ADAMTS-1 overexpression. Meanwhile, ADAMTS-1 overexpression decelerated the progress of proliferation, migration and angiogenesis in HUVECs, yet this process could be partially interrupted by treatment with PI3K and eNOS activators or overexpression of VEGF in HUVECs. To the best of our knowledge, this is the first study of the connection between ADAMTS- 1 and the PI3K/Akt-eNOSVEGF pathway in LC cells.

\section{Conclusions}

Our study demonstrated that the expression of ADAMTS-1 was decreased in A549 cells. Furthermore, overexpression of ADAMTS-1 repressed VEGF expression and A549 cell proliferation, which further inhibited the proliferation, migration and angiogenesis of HUVECs through the PI3K/ Akt-eNOS-VEGF signalling pathway. Nevertheless, we lack 
the data to validate the mechanisms of ADAMTS-1 in vivo. Further studies are needed to prove the anti-angiogenic function and potential therapeutic effect of ADAMTS-1 in LC.

\section{Acknowledgments}

Funding: This work was supported by the Zhangjiakou City Science and Technology Key Problem Project (No. 1821039D) and the Hebei Province Medical Science Research Key Project (No. 20180818).

\section{Footnote}

Conflicts of Interest: All authors have completed the ICMJE uniform disclosure form (available at http://dx.doi. org/10.21037/tcr.2019.10.34). The authors have no conflicts of interest to declare.

Ethical Statement: The authors are accountable for all aspects of the work in ensuring that questions related to the accuracy or integrity of any part of the work are appropriately investigated and resolved.

Open Access Statement: This is an Open Access article distributed in accordance with the Creative Commons Attribution-NonCommercial-NoDerivs 4.0 International License (CC BY-NC-ND 4.0), which permits the noncommercial replication and distribution of the article with the strict proviso that no changes or edits are made and the original work is properly cited (including links to both the formal publication through the relevant DOI and the license). See: https://creativecommons.org/licenses/by-nc-nd/4.0/.

\section{References}

1. Jemal A, Bray F, Center MM, et al. Global cancer statistics. CA Cancer J Clin 2011;61:69-90.

2. Ettinger DS, Akerley W, Borghaei H, et al. Non-small cell lung cancer, version 2.2013. J Natl Compr Canc Netw 2013;11:645-53.

3. Liao BC, Shao YY, Chen HM, et al. Comparative Effectiveness of First-Line Platinum-Based Chemotherapy Regimens for Advanced Lung Squamous Cell Carcinoma. Clin Lung Cancer 2015;16:137-43.

4. Abidin AZ, Garassino MC, Califano R, et al. Targeted therapies in small cell lung cancer: a review. Ther Adv Med Oncol 2010;2:25-37.
5. Popper HH. Progression and metastasis of lung cancer. Cancer Metastasis Rev 2016;35:75-91.

6. Bergers G, Benjamin LE. Angiogenesis: Tumorigenesis and the angiogenic switch. Nat Rev Cancer 2003;3:401.

7. Greenblatt M, Philippe SK. Tumor angiogenesis: transfilter diffusion studies in the hamster by the transparent chamber technique. J Natl Cancer Inst 1968;41:111.

8. Keith RL, Miller YE, Gemmill RM, et al. Angiogenic squamous dysplasia in bronchi of individuals at high risk for lung cancer. Clin Cancer Res 2000;6:1616.

9. Asahara T, Bauters C, Zheng LP, et al. Synergistic effect of vascular endothelial growth factor and basic fibroblast growth factor on angiogenesis in vivo. Circulation 1995;92:II365.

10. Mirzaaghaei S, Akrami H, Mansouri K. Ferulago angulata flower and leaf extracts inhibit angiogenesis in vitro through reducing VEGF-A and VEGFR-2 genes expression. Arch Iran Med 2014;17:278-85.

11. Cross MJ, Claesson-Welsh L. FGF and VEGF function in angiogenesis: signalling pathways, biological responses and therapeutic inhibition. Trends Pharmacol Sci 2001;22:201-7.

12. Freitas VM, Bussador DAJ, Silva TA, et al. Decreased expression of ADAMTS-1 in human breast tumors stimulates migration and invasion. Mol Cancer 2013;12:2.

13. Tang JH, Zhang HM, Zhang ZH, et al. Effect of tetramethylpyrazin combined with cisplatin on VEGF, KLF4 and ADAMTS1 in lewis lung cancer mice. Asian Pac J Trop Med 2017;10:813-8.

14. Rocks N, Paulissen G, Calvo FQ, et al. Expression of a disintegrin and metalloprotease (ADAM and ADAMTS) enzymes in human non-small-cell lung carcinomas (NSCLC). Br J Cancer 2006;94:724-30.

15. Chen J, Zhi Y, Chang X, et al. Expression of ADAMTS1 and Its Correlation with Angiogenesis in Primary Gastric Cancer and Lymph Node Metastasis. Dig Dis Sci 2013;58:405-13.

16. Banafshe HR, Hajhashemi V, Minaiyan M, et al. Iran J Basic Med Sci 2018;18:164-71.

17. Orlandini M, Galvagni F, Bardelli M, et al. The characterization of a novel monoclonal antibody against CD93 unveils a new antiangiogenic target. Oncotarget 2014;5:2750-60.

18. Jiang BH, Zheng JZ, Aoki M, et al. Phosphatidylinositol 3-kinase signaling mediates angiogenesis and expression of vascular endothelial growth factor in endothelial cells. Proc Natl Acad Sci U S A 2000;97:1749-53. 
19. Tsao AS, Scagliotti GV, Bunn JP, et al. Scientific Advances in Lung Cancer 2015. J Thorac Oncol 2016;11:613-38.

20. Weinstein N, Mendoza L, Gitler I, et al. A Network Model to Explore the Effect of the Micro-environment on Endothelial Cell Behavior during Angiogenesis. Front Physiol 2017;8:960.

21. Choi JE, Kim DS, Kim EJ, et al. Aberrant methylation of ADAMTS1 in non-small cell lung cancer. Cancer Genet Cytogenet 2008;187:80-4.

22. Lu X, Wang Q, Van Poznak C, et al. Abstract \#5667: ADAMTS1 and MMP1 promote breast cancer bone metastasis through a bone stromal cell-mediated osteolytic signaling cascade. Cancer Res 2009;69.

23. Lee NV, Sato M, Annis DS, et al. ADAMTS1 mediates the release of antiangiogenic polypeptides from TSP1 and 2. EMBO J 2006;25:5270-83.

24. Noriega-Guerra H, Cruz MC, Ribeiro PRL, et al. ADAMTS-1 disrupts HGF/c-MET signaling and HGFstimulated cellular processes in fibrosarcoma. Exp Cell Res 2018;363:271-82.

25. Vázquez F, Hastings G, Ortega MA, et al. METH1, a Human Ortholog of ADAMTS-1, and METH-2 Are Members of a New Family of Proteins with Angioinhibitory Activity. J Biol Chem 1999;274:23349-57.

26. Obika M, Ogawa H, Takahashi K, et al. Tumor growth inhibitory effect of ADAMTS1 is accompanied by the inhibition of tumor angiogenesis. Cancer Sci 2012;103:1889-97.

27. Gustavsson H, Wang W, Jennbacken K, et al. ADAMTS1, a putative anti-angiogenic factor, is decreased in human prostate cancer. BJU Int 2009;104:1786-90.

28. Li F, Huang J, Ji D, et al. Azithromycin effectively inhibits tumor angiogenesis by suppressing vascular endothelial growth factor receptor 2-mediated signaling pathways in lung cancer. Oncol Lett 2017;14:89.

29. Feliers D, Chen X, Akis N, et al. VEGF regulation of endothelial nitric oxide synthase in glomerular endothelial cells. Kidney Int 2005;68:1648-59.

30. Shiozawa T, Iyama S, Toshima S, et al. Dimethylarginine dimethylaminohydrolase 2 promotes tumor angiogenesis in lung adenocarcinoma. Virchows Archiv 2016;468:179-90.

31. Kang Z, Zhu H, Jiang W, et al. Protocatechuic acid induces angiogenesis through PI3K-Akt-eNOS-VEGF signalling pathway. Basic Clin Pharmacol Toxicol 2013;113:221.

32. Zhang R, Wang L, Zhang L, et al. Nitric Oxide Enhances Angiogenesis via the Synthesis of Vascular Endothelial Growth Factor and cGMP After Stroke in the Rat. Circ Res 2003;92:308-13.

33. Wang S, Yoon YC, Sung MJ, et al. Citrus-derived auraptene stimulates angiogenesis by activating the Erkand PI3K/Akt/eNOS-dependent signaling pathways in human umbilical vein endothelial cells. Journal of Functional Foods 2012;4:899-905.

34. Luque A, Carpizo DR, Iruelaarispe ML. ADAMTS1/ METH1 Inhibits Endothelial Cell Proliferation by Direct Binding and Sequestration of VEGF165. J Biol Chem 2003;278:23656.
Cite this article as: Wang B, Chen S, Zhao JQ, Xiang BL, Gu X, Zou F, Zhang ZH. ADAMTS-1 inhibits angiogenesis via the PI3K/Akt-eNOS-VEGF pathway in lung cancer cells. Transl Cancer Res 2019;8(8):2725-2735. doi: 10.21037/tcr.2019.10.34 